Ilias P. Doulamis / Aspasia Tzani² / Panagiotis Konstantopoulos² / Afroditi Daskalopoulou ${ }^{2}$ / Theodoros Spinos ${ }^{3}$ / Evanthia Bletsa ${ }^{3}$ / Dimitra Mitsopoulou ${ }^{3}$ / Marianna Spinou ${ }^{3}$ / Maria Evgenia Brinia ${ }^{3}$ / Konstantinos Palaiopanos ${ }^{3}$ / Laskarina Maria Korou ${ }^{2}$ / Despina N. Perrea ${ }^{2}$ / Nicholas L. Katsilambros ${ }^{2}$

\title{
Experimental hypogonadism: insulin resistance, biochemical changes and effect of testosterone substitution
}

\author{
${ }^{1}$ Laboratory for Experimental Surgery and Surgical Research "N.S. Christeas", School of Medicine, National and \\ Kapodistrian University of Athens, Athens, 15b Agiou Thoma St., Athens 11527, Greece, Phone: (617)-470-0917, E-mail: \\ doulamis.i@gmail.com. https://orcid.org/0000-0002-4128-7111. \\ ${ }^{2}$ Laboratory for Experimental Surgery and Surgical Research “N.S. Christeas”, School of Medicine, National and Kapodistrian \\ University of Athens, Athens, Greece \\ ${ }^{3}$ Athens Medical School, National and Kapodistrian University of Athens, Athens, Greece
}

\begin{abstract}
:
Background: We sought to clarify the role of testosterone substitution in terms of insulin resistance and metabolic profile dysregulation in hypogonadism.

Methods: Twenty-nine male Wistar rats aged 11-12 weeks were divided in three groups: control $(C, n=10)$, sham operation; orchiectomy (ORX, $n=9)$; and orchiectomy + testosterone substitution $(\mathrm{ORX}+\mathrm{T}, \mathrm{n}=10)$. Blood samples were obtained at day 1 (operation), after 10 days (intramuscular T injection $100 \mu \mathrm{g} / 100 \mathrm{~g}$ b.w.), 25 days (second $\mathrm{T}$ injection) and 40 days (sacrifice).

Results: Hormonal replacement significantly attenuated the negative effect of orchiectomy on insulin resistance as indicated by the successive changes in both insulin levels $(1.44 \pm 2.94$ vs. $4.10 \pm 2.47$ vs. $1.78 \pm 0.68 \mathrm{ng} / \mathrm{mL}$, for D1, D10 and D40, respectively; $\mathrm{p}=0.028$ and $\mathrm{p}=0.022$, respectively) and HOMA-IR index (1.36 \pm 2.75 vs. $3.68 \pm 1.87$ vs. $1.74 \pm 0.69 \mathrm{ng} / \mathrm{mL}$, for $\mathrm{D} 1, \mathrm{D} 10$ and $\mathrm{D} 40$, respectively; $\mathrm{p}=0.024$ and $\mathrm{p}=0.026$, respectively) in the ORX+T group. Irisin levels peaked at the 10th postoperative day and were decreased at the end of the experiment $(0.27 \pm 0.11$ vs. $0.85 \pm 0.54$ vs. $0.02 \pm 0.07 \mathrm{ng} / \mathrm{mL}$ for D1, D10 and D40, respectively; $\mathrm{p}=0.028$ in both cases), whereas resistin levels did not differ.

Experimental hypogonadism results in an unfavorable lipid profile and insulin resistance, which is not observed when the ORX animals are substituted for T.

Keywords: insulin resistance, HOMA-IR, hypogonadism, testosterone, hormonal replacement, irisin, resistin DOI: $10.1515 / \mathrm{jbcpp}-2018-0118$

Received: July 5, 2018; Accepted: January 16, 2019

\section{Introduction}

Late onset hypogonadism - a decline in the plasma concentrations of androgens - is a clinical condition that can occur naturally in aging males because the production of testosterone by the testicles begins to impair after the age of 40 years [1]. However, hypogonadism can also be iatrogenically induced for the treatment of prostate or testicular cancer [2]. Although a positive correlation between decreased circulating levels of testosterone and insulin resistance is well established, the effect of hormonal substitution in hypogonadal males with respect to insulin sensitivity is still debatable [1]. Current evidence supports that fluctuation of testosterone levels within the normal range is required in terms of the minimization of possible complications and comorbidities because both excess (e.g. steroid abuse) and deficiency of testosterone are associated with adverse effects on cardiometabolic health and insulin signaling [3].

Adipokines - such as resistin - have been extendedly studied in the pathophysiology of insulin resistance. Yet the emerging role of myokines - especially this of irisin - in insulin metabolic pathways is currently a controversial topic of research. Irisin has been proposed to be associated with increased insulin sensitivity; however, consensus has not been reached.
\end{abstract}


Given the aforementioned controversy, we sought to investigate the effect of testosterone substitution on insulin resistance and the hormonal and metabolic profile of hypogonadal male rats.

\section{Materials and methods}

\section{Animals - study design}

Male Wistar rats, 11-12 weeks old and 220-250 g in weight, were used. The animals were housed in the Laboratory for Experimental Surgery and Surgical Research, Athens University Medical School, in a controlled environment at $20 \pm 2{ }^{\circ} \mathrm{C}$, in cages compliant with the European standards (Tecniplast, West Chester, PA, USA), with $55 \%$ relative humidity, central ventilation (15 air changes/hour), and an artificial 12-h light-12-h dark cycle. Normal chow diet and water were provided ad libitum. The experimental protocol was approved by the competent Veterinary Directorate of Attica Region and was in compliance with the EU legislation (Directive 2010/63/EU) regarding the use of animals in biomedical science.

Twenty-nine rats were randomized into three groups: control $(n=10)$, in which animals underwent sham surgery; orchiectomy (ORX, $n=9)$, in which animals underwent orchiectomy; and orchiectomy and testosterone $(\mathrm{ORX}+\mathrm{T}, \mathrm{n}=10)$, in which animals underwent orchiectomy and received an intramuscular injection of testosterone enanthate/Norma (Norma Hellas S.A., Athens, Greece) at a dose of $100 \mu \mathrm{g} / 100 \mathrm{~g} / \mathrm{b} . w .10$ and 25 days, postoperatively.

Animals were euthanized at the 40th postoperative day. All animals received humane care in compliance with the "Guide for the Care and Use of Laboratory Animals" [4].

\section{Surgical procedure}

Rats were anesthetized with xylazine $(10 \mathrm{mg} / \mathrm{kg})$ and ketamine $(70 \mathrm{mg} / \mathrm{kg})$ through intramuscular injection. A 1-cm long midline incision was performed in the scrotum. Testicles were sequentially exposed and removed after the transection of spermatic cords. In sham controls, the testicles were visualized but not removed.

\section{Blood collection - biochemical measurements}

Blood samples were collected (at 9:00 AM, after a 12-h fast) at baseline (day of surgery, D1), 10 days (D10), 25 days (D25) and 40 days (D40) postoperatively using capillary tubes introduced into the medial retro-orbital venous plexus under light ether anesthesia. The serum was separated by centrifugation at $3000 \mathrm{rpm}$ for $10 \mathrm{~min}$.

Glucose (GLU), total cholesterol (CHOL), high-density lipoprotein cholesterol (HDL) and triglycerides (TG) were determined in serum, and low-density lipoprotein (LDL) was calculated by the Friedewald formula.

\section{Hormonal measurements}

Insulin, irisin and resistin levels were determined by enzyme-linked immunosorbent assay (ELISA) via commercially available kits from Elabscience Biotechnology, Co., Ltd. (Wuhan, China). All kits used the sandwich ELISA principle, and the optical density (OD) was measured spectrophotometrically at a wavelength of $450 \pm$ $2 \mathrm{~nm}$ with the use of the Bio-Rad 680 microplate reader.

Homeostatic model assessment of $\beta$-cell function and insulin resistance (HOMA-IR) was calculated as follows: glucose $(\mathrm{mg} / \mathrm{dL}) \times$ insulin $(\mathrm{mIU} / \mathrm{L}) / 405$.

\section{Statistical analysis}

Continuous variables following normal distribution are expressed as mean \pm standard deviation, and those not following normal distribution are expressed as median \pm interquartile range.

Comparisons between more than two groups were performed with analysis of variance followed by Bonferroni correction. The Kruskal-Wallis test was used as a non-parametric test for multiple group comparisons, and the Mann-Whitney U test was used for post hoc multiple testing. Comparisons between multiple time points 
for non-normal distributions were performed using Friedman test with Wilcoxon signed rank test for post hoc comparisons.

All tests were two-sided. Differences were considered as statistically significant if the null hypothesis could be rejected with $>95 \%$ confidence interval $(\mathrm{p}<0.05)$.

All statistical analyses were conducted in SPSS (version 23 for Mac OS; SPSS, Inc., Chicago, IL, USA).

\section{Results}

\section{Lipidemic and glycemic profile alterations}

At baseline (day 1), there were no difference in CHOL, HDL, LDL, TG, GLUC and body weight among groups. Orchiectomy led to an increase in serum levels of CHOL (117.50 \pm 17.50 vs. $97.50 \pm 8.75 \mathrm{mg} / \mathrm{dL}$ for ORX and control groups, respectively) and LDL (42.50 \pm 14.25 vs. $29.00 \pm 13.25 \mathrm{mg} / \mathrm{dL}$ for ORX and control groups, respectively) at the end of the experiment ( $\mathrm{p}=0.006$ and $\mathrm{p}=0.018$ for CHOL and LDL, respectively). Hormonal replacement was associated with amelioration of both parameters (CHOL $92.50 \pm 30.00 \mathrm{mg} / \mathrm{dL}$ and LDL 27.50 $\pm 12.75 \mathrm{mg} / \mathrm{dL}$ for the ORX+T group, $\mathrm{p}=0.001$ as compared with the ORX group, in both cases). No significant differences were observed for any of the other biochemical indices or body weight among groups at any time point.

\section{Insulin resistance and longitudinal hormonal changes}

Although insulin levels and HOMA-IR did not differ between the ORX and the ORX+T groups, they exhibited changes throughout the duration of the study in the ORX+T group (Figure 1). Namely, orchiectomy in this group induced an increase in insulin 10 days postoperatively $(4.10 \pm 2.47 \mathrm{vs} .1 .44 \pm 2.94 \mathrm{ng} / \mathrm{mL}, \mathrm{p}=0.028$, for D10 and D1, respectively), which was reversed with the administration of testosterone at the end of the experiment $(1.78 \pm 0.68 \mathrm{ng} / \mathrm{mL}$ for $\mathrm{D} 40, \mathrm{p}=0.022$ as compared with D10). A similar trend was also noted in the case of HOMA-IR $(1.36 \pm 2.75$ vs. $3.68 \pm 1.87$ vs. $1.74 \pm 0.69 \mathrm{ng} / \mathrm{mL}$, for D1, D10 and D40, respectively, $\mathrm{p}$ $=0.024$ and $\mathrm{p}=0.026$, respectively). Resistin levels were lower in the ORX group compared with the ORX+T group at baseline $(0.28 \pm 0.26$ vs. $1.95 \pm 0.62 \mathrm{ng} / \mathrm{mL}, \mathrm{p}=0.030$ for ORX and ORX+T groups, respectively); however, significant changes were not observed at any other time point. As far as irisin is concerned, significant differences were also shown in the ORX+T group through time. More specifically, irisin levels peaked at the 10th postoperative day and were decreased at the end of the experiment $(0.27 \pm 0.11$ vs. $0.85 \pm 0.54$ vs. $0.02 \pm$ $0.07 \mathrm{ng} / \mathrm{mL}$ for D1, D10 and D40, respectively, $\mathrm{p}=0.028$ in both cases).
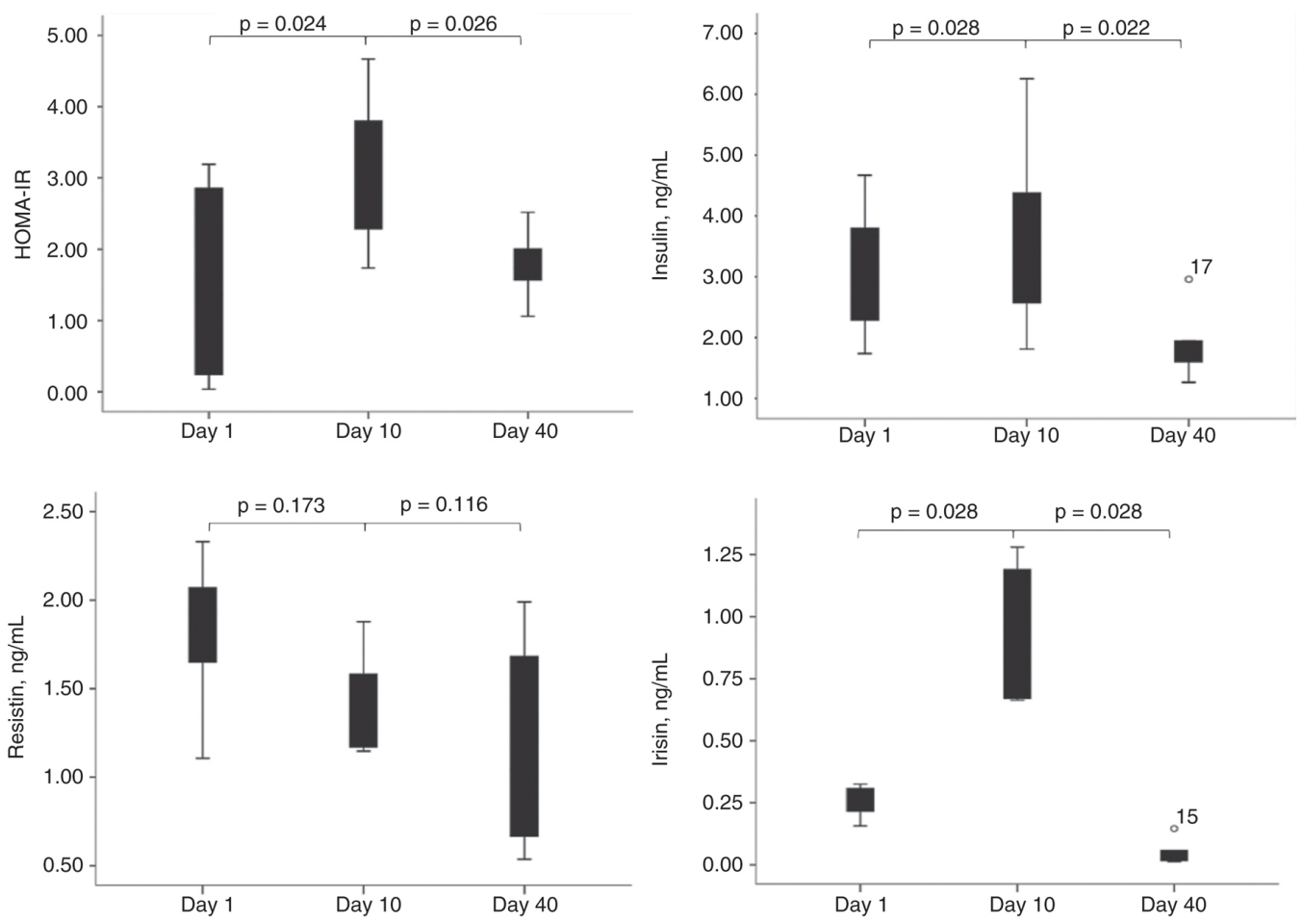
Figure 1: Box plots indicating fluctuations of HOMA-IR, insulin, resistin and irisin of the ORX+T group throughout the experiment. The $p$ values illustrate the levels of significance between D1-D10 and D10-D40. HOMA-IR, homeostatic model assessment of $\beta$-cell function and insulin resistance.

\section{Discussion}

Our findings support the existence of a protective role of testosterone replacement therapy against hypogonadism-induced insulin resistance in male rats. The respective beneficial action of hormonal replacement was also noted in the case of total and LDL cholesterol.

The interplay between low testosterone and impaired glucose metabolism has been documented but is not fully elucidated [1], [3], [5]. A causative relationship has not yet been established, and the role of interrelated confounding factors remains a possibility. A study in orchiectomized rats demonstrated that physiological doses of testosterone were associated with ameliorated insulin sensitivity whereas higher doses were not [5]. Studies in humans exhibit a heterogeneity as it concerns both the administered doses of testosterone, ranging from 10 to $300 \mathrm{mg}$, as well as the effect of hormonal replacement on insulin sensitivity [3], [6], [7].

As far as lipid metabolism is concerned, the field remains obscure because there is still a discrepancy regarding the association of androgens and lipid levels most probably due to the confounding effects of the coexistent insulin resistance [8], [9].

Irisin is a myokine that has been suggested to lead to the activation of the AMP-activated protein kinase (AMPK) pathway and increased translocation of GLUT-4, thus inducing glucose intake in myocytes and ameliorating insulin sensitivity [10]. In the present experiments, irisin levels were increased after orchiectomy and decreased after T substitution. This finding, in contrast to our expectations, was difficult to interpret. Yet this finding is in accordance with data from humans showing an inverse correlation between irisin and testosterone as well as high irisin levels in hypogonadism [11]. It has also been reported that irisin levels increase after oophorectomy, but they remain unchanged after orchiectomy [12].

Resistin levels - despite its well-established association with insulin resistance - remained unchanged despite other signs of postorchiectomy insulin resistance. We hypothesize that this could be due to relative "insensitivity" of the resistin receptors rather to an increase of resistin.

\section{Conclusion}

Orchiectomy-induced hypogonadism is associated with insulin resistance, which is partially reversed after hormonal replacement. Orchiectomized rats treated with testosterone exhibit lower levels of total and LDL cholesterol than the untreated ones, suggesting amelioration of the metabolic dysregulation in general.

Author contributions: All the authors have accepted responsibility for the entire content of this submitted manuscript and approved submission.

Research funding: None declared.

Employment or leadership: None declared.

Honorarium: None declared.

Competing interests: The funding organization(s) played no role in the study design; in the collection, analysis and interpretation of data; in the writing of the report; or in the decision to submit the report for publication.

Conflict of interests: The authors declared no conflicts of interest with respect to the research, authorship and/or publication of this article.

\section{References}

[1] Alexandersen P, Christiansen C. The aging male: testosterone deficiency and testosterone replacement. An up-date. Atherosclerosis 2004;173:157-69. 
[2] Moorjani S, Dupont A, Labrie F, Lupien P], Gagné C, Brun D, et al. Changes in plasma lipoproteins during various androgen suppression therapies in men with prostatic carcinoma: effects of orchiectomy, estrogen, and combination treatment with luteinizing hormonereleasing hormone agonist and flutamide. J Clin Endocrinol Metab 1988;66:314-22.

[3] Kapoor D, Coodwin E, Channer KS, Jones TH. Testosterone replacement therapy improves insulin resistance, glycaemic control, visceral adiposity and hypercholesterolaemia in hypogonadal men with type 2 diabetes. Eur ] Endocrinol 2006;154:899-906.

[4] Clark JD, Gebhart GF, Conder JC, Keeling ME, Kohn DF. Special report: the 1996 guide for the care and use of laboratory animals. ILAR ]. 1997:38:41-48.

[5] Holmäng A, Björntorp P. The effects of testosterone on insulin sensitivity in male rats. Acta Physiol Scand 1992;146:505-10.

[6] Friedl KE, Jones RE, Hannan C], Plymate SR. The administration of pharmacological doses of testosterone or 19-nortestosterone to normal men is not associated with increased insulin secretion or impaired glucose tolerance. J Clin Endocrinol Metab 1989;68:971-5.

[7] Mårin P, Lönn L, Andersson B, Odén B, Olbe L, Bengtsson BA, et al. Assimilation of triglycerides in subcutaneous and intraabdominal adipose tissues in vivo in men: effects of testosterone. J Clin Endocrinol Metab 1996;81:1018-22.

[8] Snyder P], Peachey H, Berlin JA, Rader D, Usher D, Loh L, et al. Effect of transdermal testosterone treatment on serum lipid and apolipoprotein levels in men more than 65 years of age. Am J Med 2001;111:255-60.

[9] Conway A], Boylan LM, Howe C, Ross G, Handelsman D]. Randomized clinical trial of testosterone replacement therapy in hypogonadal men. Int ] Androl 1988;11:247-64.

[10] Perakakis N, Triantafyllou CA, Fernández-Real ]M, Huh JY, Park KH, Seufert ], et al. Physiology and role of irisin in glucose homeostasis. Nat Rev Endocrinol 2017;13:324-37.

[11] Kamenov Z, Assyov Y, Angelova P, Gateva A, Tsakova A. Irisin and testosterone in men with metabolic syndrome. Horm Metab Res 2017;49:755-9.

[12] Zügel M, Qiu S, Laszlo R, Bosnyák E, Weigt C, Müller D, et al. The role of sex, adiposity, and gonadectomy in the regulation of irisin secretion. Endocrine 2016;54:101-10. 